\title{
Thermoelectric Shape Memory Alloy Actuators and the Issue of Thermomechanical Coupling
}

\author{
A. Bhattacharyya and D.C. Lagoudas* \\ Department of Mechanical Engineering, 5-8C Mechanical Engineering Building, University of Alberta, \\ Edmonton, Alberta T6G 2G8, Canada \\ * Department of Aerospace Engineering, 701 H.R. Bright Building, Texas A\&M University, \\ College Station; Texas 77843-3141, U.S.A.
}

\begin{abstract}
In this work, we model a thermoelectric shape memory alloy(SMA) actuator as part of a research effort to design high-force, high-frequency SMA actuators. Cyclic phase transformation in a SMA layer is achieved by alternately heating/cooling using the thermoelectric Peltier effect. The thermal and mechanical fields during phase transformation are, in general, highly coupled and the extent of this coupling in the light of different mechanical boundary conditions is examined. The primary parameters of design interest are the frequency response and the evolution of the actuation stress. The analysis resulting from the formulated boundary value problem indicates that thin SMA layers $(\approx 6 \mu$ thick), under partial transformation, are capable of delivering frequencies of about $30 \mathrm{~Hz}$ at peak stresses of about $145 \mathrm{Mpa}$.
\end{abstract}

\section{INTRODUCTION}

The phase transformation in shape memory alloys(SMAs) is usually accompanied by stresses and strains, which are quite large when compared to those observed in other advanced materials like piezoelectrics, magnetostrictive or ferroelectric materials, magnetorheological fluids etc.[9]. This is especially true for a Nickel-Titanium(Ni-Ti) SMA. Due to the aforementioned properties, SMAs are ideally suited for the design of high-force, high-displacement actuators. In applications where a moderately high actuation frequency is also a requirement(around $30-40 \mathrm{~Hz}$, for example, in rotorcraft applications), SMAs suffer from the disadvantage that their frequency response is usually low. This is because of the significant exchange of latent heat with the environment during phase transformation. Since the rate of transformation is controlled solely by the rate of heat transfer between the SMA and its environment, the heat transfer mechanisms that are employed directly control the frequency of the actuator.
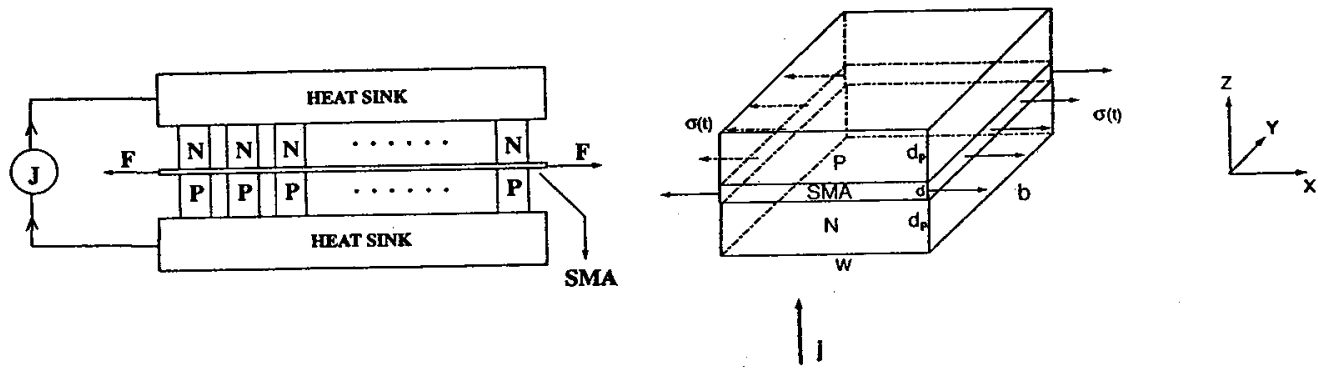

Fig. 1. (a) A conceptual SMA thermoelectric actuator, and (b) an unit cell.

Conventional heat exchange mechanisms,e.g.resistive heating and especially forced convection cooling, have been found to be quite slow in terms of actuator frequency response[2]. To address this issue, Bhattacharyya et.al.[1] proposed the use of the thermoelectric Peltier effect of semiconductors to alternately heat and cool the SMA. Such a conceptual SMA thermoelectric actuator is shown in Fig.1a, and an unit cell of such an actuator is shown in Fig.1b. A plate of SMA is used as a junction 
by sandwiching it between a pair of positively doped(P) and negatively doped(N) semiconductors. The other junction can be collectively represented as heat sinks(not shown) positioned at the ends of the $\mathrm{P}$ and $\mathrm{N}$ elements. If the SMA layer is mechanically constrained at the sides and the phase transformation is triggered on heating, an actuating stress, $\sigma(t)$, is produced. The boundary value problem(BVP) with purely thermal phase transformation(no mechanical constraints on SMA and hence no stress) was addressed by Bhattacharyya et.al.[1]. With a view towards analytically determining bounds to the thermal response, a somewhat simplified version of the same problem was addressed by Lagoudas and Ding[6]. In this paper, we extend Lagoudas and Ding's model to include the effect of the mechanical loading. Noting that the coupling between the thermal and mechanical fields is usually quite significant during phase transformation[4], the issue of thermomechanical coupling, especially in the context of different mechanical boundary conditions, is addressed. The evolution of the actuation stress, $\sigma(t)$, and the frequency response of the actuator are of interest. Other related issues of interest,e.g.actuator work output and energy efficiencies, are beyond the scope of this communication; these have been addressed elsewhere[7].

The paper is organized in the following manner. Sec.2 presents the SMA constitutive equations, Sec. 3 gives the thermomechanical model of the SMA layer and Sec. 4 gives the thermoelectric model of the P/N semiconductors. Sec.5 summarizes the analytical solution and gives some preliminary numerical results.

\section{THE CONSTITUTIVE EQUATIONS OF THẸ SMA}

It is assumed that the SMA is thermoelastic. There are several constitutive models which have been proposed to characterize the SMA thermomechanical response during phase transformation(see [8] for a discussion). For the present work, we shall use the phenomenological constitutive theory proposed by Lagoudas,Bo and Bhattacharyya[8] wherein they modeled the gradual transformation behavior of the SMA polycrystals; the salient features of the theory will be recalled. They gave the SMA response in a rate form. For simplicity(and for later use), we only give the uniaxial component, $\dot{\varepsilon}$, of the strain rate tensor corresponding to an uniaxial stress rate, $\dot{\sigma}$, on the SMA. It is

$$
\dot{\varepsilon}=\mathrm{E}_{\mathrm{T}} \dot{\sigma}+\alpha_{\mathrm{T}}^{\text {th }} \dot{\mathrm{T}}
$$

where $\mathrm{E}_{\mathrm{T}} \equiv \mathrm{E}_{\mathrm{T}}(\xi, \sigma, \mathrm{T})$ is the tangent Young's modulus and is a function of the martensitic volume fraction,$\xi$, the uniaxial stress, $\sigma$, and the temperature, T. The parameter $\alpha_{T}^{\text {th }} \equiv \alpha_{T}^{\text {th }}(\xi, \sigma, T)$ is the uniaxial component of the tangent coefficient of thermal expansion tensor and $T$ is the temperature rate. Based on the second law of thermodynamics, Lagoudas et.al.[8] derived an evolution equation for the martensitic volume fraction, given as

$$
\dot{\xi}=\left[\sqrt{\rho_{\mathrm{sma}}^{\mathrm{d}} \frac{\partial \Delta \mathrm{u}(\xi)}{\partial \xi}}\right]^{-1}(\psi \dot{\mathrm{T}}+\boldsymbol{\beta} \dot{\sigma}),
$$

where $\rho_{\text {sma }}^{d}$ is the mass density of the SMA, $\Delta u(\xi)=u_{M}(\xi)-u_{A}(\xi)$ where $u_{i}(\xi)$ is the internal energy of the $i$ th phase at the reference state(corresponding to $\sigma=0$ and $\mathrm{T}=\mathrm{T}_{0} ; \mathrm{T}_{0}$ is an arbitrary reference temperature), $\psi \equiv \psi(\xi)$ and $\beta \equiv \beta(\xi, \sigma, T)$. The interested reader is referred to Lagoudas et.al.[8] for detailed expressions of $\mathrm{E}_{\mathrm{T}}, \alpha_{\mathrm{T}}^{\text {th }}, \mathrm{u}_{\mathrm{i}}(\xi), \psi$ and $\beta$.

\section{THE THERMOMECHANICAL MODEL OF THE SMA LAYER}

The mechanical BVP is now given, followed by the thermal BVP for the SMA layer in the unit cell depicted in Fig.1b. 


\subsection{The Mechanical Boundary Value Problem}

The following discussion is based on the specific geometry of the SMA layer(Fig.1b); the cartesian coordinate system is taken with its origin at the center of the SMA layer. We assume that the displacement along the $x$-axis of all points on the surfaces, $x= \pm w / 2$, is spatially uniform and the traction on these surfaces is provided by a linear spring. This condition is stated as

$$
\left|\mathrm{u}_{\mathrm{x}}\right|=\mathrm{u}_{\mathrm{s}}(\mathrm{t}) \text { and } \int \sigma \mathrm{dA}=\mathrm{k}_{\mathrm{s}}\left(\delta_{0}-\mathrm{u}_{\mathrm{s}}(\mathrm{t})\right) \text { at } \mathrm{x}= \pm \mathrm{w} / 2 \text {; }
$$

$u_{s}(t)$ is the magnitude of the displacement(along $x$-axis) of all points on the surfaces , $x= \pm w / 2$, and may be found from a solution of the problem. The symbol $\sigma$ is used to denote the component of the stress tensor along the loading direction(x-axis), the parameter $\mathrm{k}_{\mathrm{s}}$ is a spring constant and $\delta_{0}$ is the initial stretch in the spring. The remaining surfaces are taken to be traction-free for simplicity. It is then easy to show that, in absence of body forces and inertial effects, an uniaxial stress field given by $\sigma \equiv \sigma(y, z, t)$ satisfies the assumed mechanical boundary conditions. We now note that Bhattacharyya et.al.[1]'s work on the purely thermal problem(no mechanical constraints on the SMA) revealed that the temperature gradients in a Ni-Ti SMA was practically negligible because of its high thermal diffusivity. Therefore, we assume a spatially uniform temperature field in the SMA,i.e. $T \equiv T(t)$. Further, assuming an initially fully martensitic $\operatorname{SMA}(i . e . \quad \xi=1$ at $t=0$ ), we can infer from Eq.2 that $\dot{\xi} \equiv \dot{\xi}(\mathrm{y}, \mathrm{z}, \mathrm{t})$ and therefore, from Eq.1, $\dot{\varepsilon} \equiv \dot{\varepsilon}(\mathrm{y}, \mathrm{z}, \mathrm{t})$. This latter relation, in the context of small strains, implies $\dot{\mathrm{u}}_{\mathrm{x}}=\dot{\varepsilon} \mathrm{x}$ and due to the displacement boundary condition of Eq.3, $\dot{\varepsilon}$ turns out to be spatially uniform,i.e. $\dot{\varepsilon} \equiv \dot{\varepsilon}(\mathrm{t})$. For this to be valid, it may be inferred from Eq.1 that the stress is also spatially uniform,i.e. $\sigma \equiv \sigma(t)$. The spatially uniform stress follows from the traction boundary condition of Eq. 3 as

$$
\sigma(t)=\frac{k_{s}}{b d}\left(\delta_{0}-u_{s}(t)\right),
$$

where $b$ and $d$ are dimensional parameters of the SMA. With the stress and temperature being spatially uniform, Eq.2 points to the fact that $\xi$ is also spatially uniform. The values of the spatially uniform martensitic volume fraction, stress and temperature at $\mathrm{t}=0$ are summarized as

$$
\xi(0)=1 \quad, \quad \sigma(0)=\frac{k_{s} \delta_{0}}{b d+k_{s} w /\left(2 E_{M}\right)} \quad, \quad T(0)=T_{0} .
$$

The second equation in the above follows from the total form of Eq. 1 with $T=0$ (resulting in the Hooke's Law), Eq.4 and the relation $u_{x}=\varepsilon x$. Note that the parameter $E_{M}$ is the Young's modulus of martensite. Finally, the rate form of Eq.4 alongwith Eq.1 gives the stress rate directly in terms of the temperature rate

$$
\dot{\sigma}(\mathrm{t})=s(\xi, \sigma, \mathrm{T}) \dot{\mathrm{T}}(\mathrm{t}) \quad \text { with } s(\xi, \sigma, \mathrm{T})=-\left[\frac{2 \mathrm{bd}}{\mathrm{w}} \frac{\mathrm{E}_{\mathrm{T}}(\xi, \sigma, \mathrm{T})}{\mathrm{k}_{\mathrm{s}}}+1\right]^{-1} \mathrm{E}_{\mathrm{T}}(\xi, \sigma, \mathrm{T}) \alpha_{\mathrm{T}}^{\text {th }}(\xi, \sigma, \mathrm{T}) .
$$

The thermomechanical state of the SMA layer will be fully characterized once $\xi(t), \sigma(t)$ and $T(t)$ are known. The evolution equations for the first two are given by Eqs.2 and 6; once an evolution equation for the temperature is also available, the three equations can be solved and along with the initial conditions given by Eqs.5, $\xi(\mathrm{t}), \sigma(\mathrm{t})$ and $\mathrm{T}(\mathrm{t})$ at any $\mathrm{t}>0$ may be found. The evolution equation for the temperature is now developed.

\subsection{The Thermal Boundary Value Problem}

The thermal boundary conditions for the SMA are now given. We write

$$
\overrightarrow{\mathrm{q}} \cdot \hat{\mathrm{n}}=-\mathrm{h}\left(\mathrm{T}-\mathrm{T}_{0}\right) \text { on } \mathrm{x}= \pm \frac{\mathrm{w}}{2}, \mathrm{y}= \pm \frac{\mathrm{b}}{2} \text { and } \overrightarrow{\mathrm{q}} \cdot \hat{n_{i}}=\mathrm{q}_{\mathrm{i}} \text { on } \mathrm{S}_{\mathrm{I}}: \mathrm{z}= \pm \frac{\mathrm{d}}{2} \text {, }
$$


where $\overrightarrow{\mathbf{q}}$ is the heat flux vector, $\hat{\mathbf{n}}$ is an outward unit normal on the SMA surface, the parameter $\mathrm{h}$ is a convection coefficient and $T_{0}$ is the ambient temperature. With regard to the second condition, $\hat{n}_{p}=-\hat{n}_{n}=\hat{e}_{z}$, where $\hat{e}_{z}$ is an unit vector along the positive direction of the $z$-axis. The parameter $\mathrm{q}_{\mathrm{i}}$ (a scalar quantity) will come from the interface condition between the SMA and the P/N; it will be given in the next section. An equation of energy balance can be written for the SMA, and incorporating the thermal boundary conditions(Eq.7), we have

$$
-\frac{1}{V_{s m a}} \int_{S_{I}}\left(q_{n}+q_{p}\right) d S-2 h\left(\frac{1}{b}+\frac{1}{w}\right)\left(T(t)-T_{0}\right)+\rho J(t)^{2}=C(\xi) \dot{T}+\alpha^{\text {th }}(\xi) T(t) \dot{\alpha}(t)+\gamma(\sigma, T) \dot{\xi}
$$

where $V_{s m a}$ is the SMA volume, $S_{I}$ has been defined in Eq.7, $\rho$ is the electrical SMA resistivity, $J(t)$ is the magnitude of the current density, $C(\xi)$ is the heat capacity of the SMA, $\alpha^{\text {th }}(\xi)$ is the coefficient of thermal expansion and $\gamma(\sigma, T)$ is a parameter(refer Lagoudas et.al.[8] for details). While $\dot{\xi}$ follows from Eq. 2 during phase transformation, Eq. 8 reduces to the usual heat conduction equation for a thermoelastic material[3] in absence of phase transformation(i.e.when $\dot{\xi}=0$ ). In the next section, we define the terms $q_{n}$ and $q_{p}$ (see first term in Eq.8).

\section{THE THERMOELECTRIC PROBLEM OF THE P/N SEMICONDUCTORS}

We shall assume for simplicity that the $\mathrm{P} / \mathrm{N}$ semiconductors are stress-free. Thus, only the thermoelectric problem of the semiconductors need to be addressed. We begin by assuming that the temperature has a gradient only in the $z$-direction[1], thus $T \equiv T_{i}(z, t)$ where $i=P, N$, An isothermal boundary condition(representing heat sinks) is assumed at the end surfaces, while the temperatures at the SMA/P and SMA/N interfaces are taken to be continuous. These conditions are stated respectively as

$$
T_{i}\left( \pm d / 2 \pm d_{p}, t\right)=T_{0} \quad \text { and } \quad T(t)=T( \pm d / 2, t) \quad(i=P, N) .
$$

The heat flux at the interfaces, however, has a jump due to the Peltier effect of the P/N. It is

$$
\left(\vec{q}-\vec{q}_{i}\right) \cdot \hat{n}_{i}=\alpha, T(t) \vec{J} \cdot \hat{n}_{i} \quad(i=P, N),
$$

where $\vec{q}_{i}$ is the heat flux vector and $\alpha_{i}$ is the Seebeck coefficient corresponding to the $i$ th phase. Note that the term $\alpha_{i} \mathbf{T}(t) \vec{J} . \hat{n}_{i}$ may be viewed as a heat source/sink at the considered interface, and represents the Peltier effect. This phenomenon is controlled by the Seebeck coefficient, $\alpha_{i}$, which owes its name to the thermoelectric Seebeck effect(Peltier and Seebeck effects are different manifestations of the same underlying phenomenon[5]). Higher is the current density, stronger is the Peltier effect. However, beyond a certain critical value[6], the joule heating dominates the Peltier effect. The initial condition for the temperature is assumed simply as

$$
\mathrm{T}_{\mathrm{i}}(\mathrm{z}, \mathrm{t})=\mathrm{T}_{0} \quad(\mathrm{i}=\mathrm{P}, \mathrm{N}) .
$$

The heat conduction equation for the semiconductor elements is[6]

$$
k_{i} \frac{\partial^{2} T_{i}}{\partial z^{2}}(z, t)-2 h\left(\frac{1}{b}+\frac{1}{w}\right)\left(T_{i}(z, t)-T_{0}\right)+p_{i} J(t)^{2}=C_{i} \frac{\partial T_{i}}{\partial t}(z, t), \quad(i=P, N), \quad t>0,
$$

where $k_{i}, \rho_{i}$ and $C_{i}$ are the thermal conductivity, the electrical resistivity and the heat capacity of the $i$ th phase respectively. It can be shown(on the same lines as the purely thermal problem[6]) that when $k_{p}=k_{N}, p_{p}=\rho_{N}, C_{p}=C_{N}$ and $\alpha_{p}=-\alpha_{N}$ (this last condition maximizes the Peltier effect[1]), the temperature distribution is symmetrical about the plane, $z=0$,i.e. $T_{p}(z, t)=T_{N}(-z, t)$. It can also be shown that the terms $\mathrm{q}_{\mathrm{n}}$ and $\mathrm{q}_{\mathrm{p}}$ in Eq.8 maybe derived from Eq.10 to result in

$$
q_{n}=q_{p}=-k_{p} \frac{\partial T_{p}}{\partial z}(z, t)+\alpha_{p} T(t) J(t)
$$


Eqs.5 and 11, for $\xi(t), \sigma(t), T(t)$ and $T_{p}(z, t)$ respectively. The solution of the latter two equations(at a given $\xi$ and $\sigma$ ) follow next.

\section{THE ANALYTICAL SOLUTION AND NUMERICAL RESULTS}

\subsection{The Analytical Solution}

Lagoudas and Ding[6] solved Eqs.8 and 12(in absence of a stress field) by reformulating the problem as an integro-differential equation solely in the temperature of the SMA; the temperatures of the P and $\mathrm{N}$ are not involved. For the present problem(with the mechanical field), an identical procedure is followed. Thus the following differential equation in terms of the SMA temperature results

$$
\frac{d T}{d t}(t)=S(t)-v_{1} T(t), \quad t>0,
$$

where $T(t)$ can be solved from the above equation once $S(t)$ follows from the solution of the following integro-differential equation

$$
\int_{0}^{t} G(t-\tau) S(\tau) d \tau+\mu(\xi, \sigma, T) S(t)+\left[v_{2}(t)-\mu(\xi, \sigma, T) v_{1}\right] \int_{0}^{t} e^{-v_{1}(t-\tau)} S(\tau) d \tau=F(t)-T_{0}\left[v_{2}(t)-\mu(\xi, \sigma, T) v_{1}\right] e^{-v_{1} t}
$$

The parameters $G(t), v_{2}(t), v_{1}$ and $F(t)$ are identical to those in [6] while the parameter $\mu(\xi, \sigma, T)$ is given in [7]. A simultaneous solution of Eqs.14 and 15 with Eqs.2 and 6 may be accomplished by the fourth-order Runge Kutta method. In addition, at every time step, an iterative procedure is implemented as the coefficients of the evolution equations themselves depend on $\xi, \sigma$ and $\mathrm{T}$; the interested reader is referred to [7] for details.

\subsection{Numerical Results}

While the material properties of a Ni-Ti SMA and P/N semiconductors have been given in [7], it is worthwhile mentioning some of the important parameters used in the numerical results. The stressfree martensitic start and finish temperatures have been taken as $23^{\circ} \mathrm{C}$ and $1^{\circ} \mathrm{C}$ respectively, whereas the stress-free austenitic start and finish temperatures have been taken as $29^{\circ} \mathrm{C}$ and $51^{\circ} \mathrm{C}$. The geometric parameters used for the actuator are: $b=w=4 \mathrm{~mm}, d_{p}=2 \mathrm{~mm}$ and $d=0.5 \mathrm{~mm}$. The initial conditions(refer Eq.5) are taken as $\delta_{0}=0$ and $T_{0}=297 \mathrm{~K}$. We assume $\mathrm{k}_{\mathrm{s}}=3 \mathrm{KN} / \mathrm{mm}$ and $\mathrm{J}(\mathrm{t})=3$ Amps $/ \mathrm{mm}^{2}$.

We shall now examine the issue of thermomechanical coupling. With reference to Eq.8, it may be noted that, in absence of phase transformation(i.e. $\dot{\xi}=0$ ), the thermomechanical coupling is through the stress rate term on the right hand side. However, as long as the loading is quasistatic(to which we restrict this study), the coupling term is found to be about two orders of magnitude smaller than the temperature rate term. In fact, the coupling term may be dropped from the heat equation without appreciable loss in accuracy, leading to the well-known decoupling of the thermal and mechanical fields[3]. However, during phase transformation, an additional thermomechanical coupling term is

operative in Eq. 8 because $\xi$ itself depends on the stress rate(see Eq.2). This coupling term is found to be of the same order of magnitude as the temperature rate term and therefore may not be dropped from the heat equation. We also note that the mechanical boundary condition can also have a drastic effect on the thermomechanical coupling. Examining Eq.4, it is apparent that if $u_{s}(t) / \delta_{0} \ll 1$, the stress may be approximated as $\sigma(t) \approx \mathrm{k}_{\mathrm{s}} \delta_{0} /(\mathrm{bd})$, a constant value. In that case, $\dot{\sigma}(\mathrm{t}) \approx 0$ (i.e.implying a constant load boundary condition), leading to a decoupling of the thermal and mechanical fields. In fact, the implication for an initially martensitic SMA is that when it is subjected to an initial stress of $50 \mathrm{Mpa}$, two different spring stiffnesses of $3 \mathrm{KN} / \mathrm{mm}$ and $1 \times 10^{-3} \mathrm{KN} / \mathrm{mm}$ result in actuation frequencies of $1.36 \mathrm{~Hz}$ and $1.67 \mathrm{~Hz}$ respectively. It can be shown that $\delta_{0}$ is significantly higher in the latter case, thus approximating a constant load boundary condition. The resulting vanishing stress rate implies that the martensite to austenite transformation is quicker, resulting in a higher frequency. 
frequencies of $1.36 \mathrm{~Hz}$ and $1.67 \mathrm{~Hz}$ respectively. It can be shown that $\delta_{0}$ is significantly higher in the latter case, thus approximating a constant load boundary condition. The resulting vanishing stress rate implies that the martensite to austenite transformation is quicker, resulting in a higher frequency. In general, higher frequencies may be achieved with a higher current density(but less than some
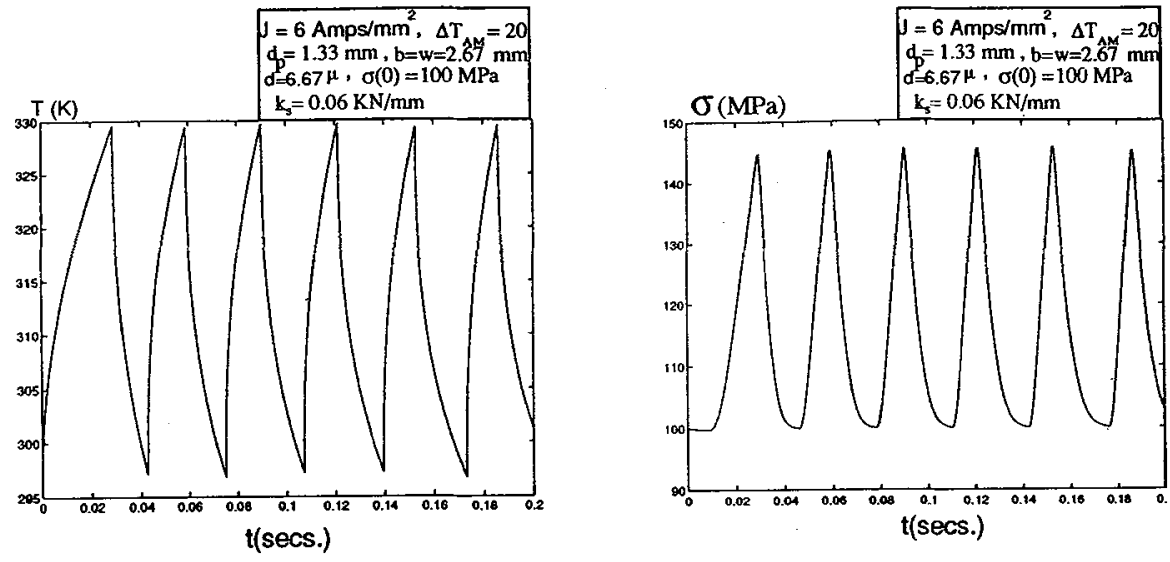

Fig. 2. The evolution of (a) temperature of SMA layer and (b) actuation stress vs. time; note $\Delta \mathrm{T}_{\mathrm{AM}}=\mathrm{A}_{\mathrm{s}}^{0}-\mathrm{M}_{\mathrm{f}}^{0}=\mathrm{A}_{\mathrm{f}}^{0}-\mathrm{M}_{\mathrm{s}}^{0}$.

critical value[6]), a lower temperature hysteresis and a thin SMA layer. Specifically, it is found that when $\mathrm{J}(\mathrm{t})=6 \mathrm{Amps} / \mathrm{mm}^{2}, \mathrm{~d}=6.67 \mu, \mathbf{A}_{\mathrm{s}}^{0}=21^{0} \mathrm{C}, \mathbf{A}_{\mathrm{f}}^{0}=43^{0} \mathrm{C}$ (with same martensitic temperatures as before), the actuator delivers peak stresses of around $145 \mathrm{Mpa}$ at a frequency of around $30.15 \mathrm{~Hz}$ during cyclic partial phase transformation between a martensitic volume fraction of $100 \%$ to $80 \%$. The evolution of the temperature in the SMA and the actuation stress is shown in Fig.2.

\section{Acknowledegments}

This work was made possible due to the financial support of the United States Office of Naval Research. through their contract No.N 00014-94-1-0268, monitored by Dr.Roshdy Barsoum.

\section{References}

[1]. Bhattacharyya,A.,Lagoudas,D.C.,Wang,Y. and Kinra,V.K.,J. Smart Mat. Struct., 4, (1995) 252.

[2]. Bo,Z. and Lagoudas,D.C.,"Deformations and Thermal Response of Active Flexible Rods with Embedded SMA Actuators", Smart Structures and Materials 1994: Smart Structures and Intelligent Systems, Orlando,Florida,2190,1994.

[3]. Boley,B.A. and Weiner,J.H.,Theory of Thermal Stresses(Krieger Pub.,Florida,1960).

[4]. Delaey,L., Krishnan, R.V., Tas,H. and Warlimont, H., J. Mat. Sci., (1974) 1521.

[5] Domenciali,C.A., Reviews of Modern Physics, 26, No.2(1954) 237.

[6]. Lagoudas,D.C. and Ding, Z., Int. J. Engng. Sci., 33, No.15(1995), 2345.

[7]. Lagoudas,D.C. and Bhattacharyya,A., "Modeling of Thin Layer Extensional Thermoelectric SMA Actuators", Int. J. Solids Struct.(submitted).

[8]. Lagoudas,D.C.,Bo.,Z. and Bhattacharyya,A.,"A Thermodynamic Constitutive Model for Gradual Phase Transformation of SMA Materials", Proceedings of the Smart Structures symposium, SPIE, San Deigo, California(1996).

[9]. Takagi,T.,J. Int. Mater. Sys. Struct., 1, (1990)149. 